\title{
FORMULAÇÕES DE ATRAZINA EM XEROGÉIS: SÍNTESE E CARACTERIZAÇÃo
}

\section{Letícia Gomes de Ávila*, Simone Benvenuti Leite e Deborah Pinheiro Dick}

Departamento de Físico-Química, Instituto de Química, Universidade Federal do Rio Grande do Sul, CP 15003, 91501-970 Porto Alegre - RS, Brasil

\section{Adriana Raffin Pohlmann}

Departamento de Química Orgânica, Instituto de Química, Universidade Federal do Rio Grande do Sul, CP 15003, 91501-970 Porto Alegre - RS, Brasil

Recebido em 16/6/08; aceito em 27/3/09; publicado na web em 10/8/09

\begin{abstract}
ATRAZINE FORMULATIONS IN XEROGELS: SYNTHESIS AND CHARACTERIZATION. In this study, controlled release formulations of Atrazine (ATZ) were synthesized by the sol-gel method and characterized by elemental, FTIR, SEM, BET and DSC analyses. The release kinetic of ATZ from the formulations in $\mathrm{CaCl}_{2} 0.01 \mathrm{~mol} \mathrm{~L}^{-1}$ medium was monitored by UV/Vis spectroscopy. In all formulations, ATZ was physically dispersed on the Si-polymer, and the dispersion grade decreased with increasing amount of herbicide. The ATZ release kinetics was controlled mainly by dissolution, and the data could be fitted to the Korsmeyer - Pepper model. The ATZ as xerogel presents a lower affinity for soil than as granulated form.
\end{abstract}

Keywords: sol-gel; sorption; soil.

\section{INTRODUÇÃO}

A atrazina ([2-cloro-4-(etilamino)-6-(isopropilamino)-1,3,5-triazina]) é um herbicida empregado principalmente nas culturas de canade-açúcar e de milho, as quais ocupam, respectivamente, o segundo e o terceiro lugar da produção anual nacional. ${ }^{1}$ Aproximadamente $17.10^{6}$ hectares do território nacional são utilizados para estas culturas, sendo as mesmas responsáveis por $36,5 \%$ do consumo anual nacional de herbicidas. ${ }^{1}$

O uso intensivo da atrazina no mundo e sua considerável mobilidade nos solos têm contribuído para que níveis acima do limite permitido sejam frequentemente detectados em águas de superfície e subterrâneas na Europa ${ }^{2,3}$ e nos Estados Unidos. ${ }^{4}$ Os limites máximos permitidos para a atrazina em águas para consumo humano são de 3 $\mu \mathrm{g} \mathrm{L}^{-1}$ nos Estados Unidos ${ }^{5}$ e $0,5 \mu \mathrm{g} \mathrm{L}^{-1}$ na Europa. O tempo de meia vida da atrazina pode variar de 2 meses a 6 anos, dependendo das condições do meio. Este herbicida apresenta vários metabólitos, que possuem diferentes graus de toxicidade e tempo de meia vida, sendo os mais comuns a hidroxiatrazina e a dietilatrazina. ${ }^{6}$

Uma molécula de herbicida ao alcançar o solo pode ser absorvida pelas plantas, manifestando seus mecanismos de ação; sofrer transformações químicas que promovem sua volatilização e decomposição fotocatalítica; ser degradada pela ação de micro-organismos ou enzimas (biodegradação); ser sorvido pelo solo ou por resíduos vegetais. ${ }^{7}$ O produto sorvido pode ser removido por percolação ou pela erosão até corpos de água adjacentes, onde pode persistir contaminando e afetando a vida aquática. Por outro lado, a sorção do herbicida no solo pode retardar ou impedir o movimento das moléculas no meio, afetando os processos de transformação e transporte, a eficiência agronômica do ativo e a contaminação do ambiente. A recomendação da dose de aplicação de um herbicida de baixa solubilidade em água, tal como a atrazina, é geralmente feita baseando-se no teor de matéria orgânica do solo, que é o seu principal sorvente. Quanto maior o teor de matéria orgânica maior a retenção do herbicida e, portanto, maior será a dose recomendada para que seja atingido o efeito biocida almejado. ${ }^{8}$

*e-mail: leticia@iq.ufrgs.br
O processo de sorção de um herbicida pode ser caracterizado quantitativamente pelo coeficiente de distribuição $K_{d}$ e pelo coeficiente de distribuição normalizado pelo teor de carbono presente no solo $\mathrm{K}_{\mathrm{oc}}$. O K $\mathrm{d}$ é obtido a partir da relação linear entre a quantidade sorvida por massa de solo $\left(\mathrm{Q}_{\mathrm{s}}\right)$ e a concentração na solução de equilíbrio $\left(\mathrm{C}_{\mathrm{e}}\right) \cdot{ }^{9}{ }^{10} \mathrm{O}$ índice $\mathrm{K}_{\mathrm{oc}}$ é determinado dividindo-se o coeficiente $\mathrm{K}_{\mathrm{d}}$ pelo teor de Carbono na amostra ( $\mathrm{g} \mathrm{C} \mathrm{kg}^{-1}$ de solo).

O desenvolvimento de sistemas de liberação controlada a partir de matrizes inertes é uma alternativa para aumentar a eficiência do ativo e reduzir eventuais efeitos tóxicos, promovendo melhor disponibilidade e direcionamento da ação. ${ }^{11}$ Estes sistemas podem carrear substâncias biologicamente ativas, alterando a distribuição do ativo e aumentando sua ação biológica.

O método sol-gel é uma técnica que oferece novas possibilidades de suportar compostos orgânicos a partir da hidrólise e policondensação de alcóxissilanos formando uma matriz sólida tridimensional que permite a produção de sistemas de liberação controlada. ${ }^{12}$ Esse método tem sido muito utilizado na síntese de materiais carreadores, pois sendo realizado à temperatura ambiente, permite o aprisionamento de moléculas orgânicas sem o risco de degradação térmica. ${ }^{13}$ As substâncias biologicamente ativas no gel ficam distribuídas na superfície e dentro dos poros da estrutura de sílica. Na síntese de xerogéis para a liberação controlada, a introdução do ativo é feita por adição ao sistema, antes da policondensação, sendo que sua solubilidade no meio aquoso limita a quantidade que pode ser adicionada durante a fase sol. ${ }^{14}$

A aplicação de herbicidas associados a sistemas carreadores, que sejam gradualmente absorvidos pela planta no intervalo de $24 \mathrm{~h}$ sem que haja forte retenção pela matriz do solo, pode representar uma alternativa para a diminuição da dose recomendada, enquanto mantém efetiva a sua atividade biológica. Desta forma, pode-se reduzir a lixiviação do xenobiótico e seus metabólitos ao longo do perfil do solo, bem como as perdas por evaporação e fotólise. ${ }^{15,16}$ Consequentemente, esta prática pode contribuir para a mitigação do impacto ambiental causado pela intensa utilização destes produtos na agricultura brasileira.

No Brasil alguns estudos que investigam herbicidas de liberação controlada empregando silício no sistema carreador, que é um elemento abundante no solo, já foram realizados. Para os pesticidas 2,4-D e diuron, imobilizados em superfície de sílica-gel tendo como 
precursor 3-trimetoxissililpropilamina, verificou-se que o grupo cloro dos pesticidas se ligava covalentemente ao grupo amina disponível do precursor. ${ }^{17,18}$ Posteriormente foi observado que o efeito tóxico do diuron na microbiota do solo diminui quando o mesmo se encontra ancorado na sílica. ${ }^{19} \mathrm{Na}$ imobilização do herbicida picloram na superfície de sílica-gel pré-funcionalizada constatou-se não apenas decréscimo da sua toxidade para a microbiota como também diminuição da velocidade de liberação em relação ao pesticida comercial. ${ }^{20}$

Considerando-se a larga utilização da atrazina no Brasil, o seu uso na agricultura na forma associada a sistemas carreadores de liberação controlada pode ser interessante tanto do ponto de vista ecológico como econômico. Portanto, o objetivo deste trabalho foi caracterizar formulações de atrazina sintetizadas pelo método sol-gel, bem como estudar sua cinética de liberação em meio aquoso e avaliar o comportamento sortivo em três tipos de solos.

\section{PARTE EXPERIMENTAL}

\section{Síntese das formulações - método sol-gel - SGATZ}

A síntese das formulações foi realizada segundo o método sol-gel, ${ }^{21}$ adaptado às condições experimentais para atrazina, empregando-se os seguintes reagentes: tetraetil-orto-silicato (TEOS) - (Acros Organics 98\%); álcool etílico 99,8\% (Nuclear grau PA); fluoreto de sódio (NaF) - (Nuclear); atrazina comercial marca Nortox 500 SC. A atrazina (ATZ), em fase sólida, foi obtida pela secagem da solução de ATZ comercial, contendo $500 \mathrm{~g} \mathrm{~L}^{-1} \mathrm{de}$ princípio ativo em suspensão, em estufa a vácuo em temperatura controlada na faixa de $20-25^{\circ} \mathrm{C}$.

Para a síntese, uma solução de catalisador foi preparada em um becker de vidro adicionando-se $0,4 \mathrm{~g}$ de $\mathrm{NaF}$ em $2 \mathrm{~mL}$ de água deionizada sob agitação magnética até completa homogeneização. $\mathrm{O}$ xerogel foi sintetizado por processo sol-gel em becker de vidro onde adicionaram-se $5 \mathrm{~mL}$ de solução de TEOS $98 \%$ e $10 \mathrm{~mL}$ de álcool etílico 99,8\%. O sistema foi submetido à agitação magnética até completa homogeneização. O herbicida ATZ foi adicionado na forma sólida nesta etapa e o sistema mantido sob agitação. A fase sol pura (SG) ou misturada com ATZ foi adicionada gota a gota ao catalisador mantido sob agitação até a formação do gel detectada visualmente pela precipitação. Quanto maior a massa adicionada de herbicida, maior foi o tempo de agitação necessário, chegando até $5 \mathrm{~h}$ nas formulações mais concentradas. Após a formação do gel, a agitação foi desativada e o solvente foi evaporado sob temperatura de $25^{\circ} \mathrm{C}$ e pressão atmosférica, em capela, até a obtenção do pó fino e livre de solvente (xerogel). Foram sintetizadas quatro formulações de ATZ que diferem quanto à massa (mg) de herbicida adicionada a cada unidade de síntese de xerogel: $150 \mathrm{mg}$ de ATZ (SGATZ150), $200 \mathrm{mg}$ de ATZ (SGATZ200), $300 \mathrm{mg}$ de ATZ (SGATZ300) e 500 mg de ATZ (SGATZ500).

\section{Caracterização das formulações e dos produtos comerciais}

Além das formulações de ATZ (SGATZ), foram também caracterizadas amostras de xerogel puro, de ATZ pura (99\% de princípio ativo, Milênia), ATZ granulada (Gesaprim GrDA Syngenta) e ATZ comercial em fase sólida utilizada na síntese das formulações (Nortox $500 \mathrm{SC}$ seca). Previamente às análises, todas as amostras foram secas por $24 \mathrm{~h}$ em estufa a vácuo e temperatura de $25^{\circ} \mathrm{C}$.

\section{Análise elementar e determinação da concentração de ATZ nas} formulações

A composição elementar das amostras foi determinada em analisador elementar Perkin Elmer 2400 em duplicata. Para o cálculo da concentração de ATZ nas formulações e nas amostras comerciais considerou-se a proporção ponderal teórica de nitrogênio presente na atrazina, que é de 32,45\%. A partir do teor de nitrogênio, calculou-se a concentração real de ATZ ([ATZ $\mathrm{F}]$ ) nas formulações pela Equação 1.

$\left[A T Z_{F}\right]=\frac{N \% x 1000}{32,45}$

onde $\left[\mathrm{ATZ}_{\mathrm{F}}\right]$ é a concentração de atrazina em $\mathrm{mg} \mathrm{g}^{-1}$ e $\mathrm{N} \%$ é o teor de nitrogênio na amostra determinado pela análise elementar.

$\mathrm{O}$ rendimento da reação $(\mathrm{R})$ foi calculado relacionando-se a concentração de $\mathrm{ATZ}\left(\mathrm{ATZ}_{\mathrm{F}}\right)$ determinada na formulação a partir da análise elementar com a concentração de ATZ (ATZ) adicionada na síntese (Equação 2).

$R(\%)=\frac{A T Z_{F}\left(m g \cdot g^{-1}\right)}{A T Z_{I}\left(m g \cdot g^{-1}\right)} x 100$

\section{Isotermas de adsorção e dessorção de nitrogênio}

Isotermas de adsorção e dessorção de nitrogênio das amostras previamente degaseificadas, foram realizadas sob vácuo a $40{ }^{\circ} \mathrm{C} \mathrm{em}$ aparato volumétrico, conectado a uma bomba de vácuo Edwards, empregando um manômetro capilar de Hg para as medidas de pressão. A partir da isoterma de adsorção calculou-se a área superficial específica dos materiais pelo método BET.

\section{Calorimetria diferencial de varredura - DSC}

A fim de investigar o grau de cristalinidade da ATZ nas formulações $^{22}$ foram realizadas análises de calorimetria diferencial de varredura (DSC), em duplicata, sob atmosfera inerte de nitrogênio, empregando-se uma taxa de aquecimento de $10{ }^{\circ} \mathrm{C} \mathrm{min}{ }^{-1}$, na faixa de 50 a $200{ }^{\circ} \mathrm{C}$ em equipamento Perkin Elmer DSC 4 Differential Scanning Calorimeter. Os valores de entalpia de fusão $(\Delta \mathrm{H})$ obtidos dos termogramas foram corrigidos pelo teor de ATZ ([ATZ]) em cada formulação de acordo com a Equação 3.

$$
\Delta H_{\text {fusão }}\left(\mathrm{cal} . \mathrm{g}^{-1} A T Z\right)=\frac{\Delta H\left(\mathrm{cal} \cdot \mathrm{g}^{-1}\right)}{[A T Z]\left(g A T Z . \mathrm{g}^{-1}\right)}
$$

\section{Microscopia eletrônica de varredura (MEV)}

As amostras foram analisadas por microscopia eletrônica de varredura (MEV) (Jeol Scanning Microscope, JSM-5800) equipada com detector de energia dispersiva de raios-X (EDS), fixadas com fita de carbono de dupla face e recobertas com ouro através de técnicas convencionais de sputtering. As análises foram realizadas no Centro de microscopia eletrônica da UFRGS, Porto Alegre, RS.

\section{Espectroscopia de infravermelho (FTIR)}

Foram realizadas análises de espectroscopia de infravermelho com transformada de Fourier (FTIR), em equipamento Shimadzu FTIR 8300, varrendo-se o espectro de 4000 a $400 \mathrm{~cm}^{-1}$, com resolução de $4 \mathrm{~cm}^{-1}, 32$ scans, empregando-se $1 \mathrm{mg}$ de amostra e $100 \mathrm{mg}$ de $\mathrm{KBr}$. O KBr foi seco previamente a $105^{\circ} \mathrm{C}$ durante $3 \mathrm{~h}$ e resfriado em dessecador por $24 \mathrm{~h}$.

\section{Liberação de ATZ das formulações em meio aquoso}

Os testes de liberação de ATZ das formulações e da ATZ granulada foram realizados em solução de $\mathrm{CaCl}_{2} 10^{-2} \mathrm{~mol} \mathrm{~L}^{-1}$ para simular a força 
iônica do solo quantificando-se a atrazina em solução pela medida da absorbância a $222 \mathrm{~nm}$ no equipamento UV-160 A Shimadzu. Previamente aos testes de liberação, foi traçado o espectro de uma solução de $15 \mathrm{mg} \mathrm{L}^{-1}$ de ATZ em meio de $\mathrm{CaCl}_{2} 10^{-2} \mathrm{~mol} \mathrm{~L}^{-1}$ na região de $200 \mathrm{a}$ $800 \mathrm{~nm}$, sendo determinada a absorbância máxima em $222 \mathrm{~nm}$.

Para a curva padrão foram preparadas soluções aquosas $\left(\mathrm{CaCl}_{2}\right.$ $10^{-2} \mathrm{~mol} \mathrm{~L}^{-1}$ ) de ATZ de $0 ; 1,64 ; 2,18 ; 2,7 ; 6$ e $12 \mathrm{mg} \mathrm{L}^{-1}$. As soluções padrão foram obtidas por diluição de uma solução de $30 \mathrm{mg} \mathrm{L}^{-1}$ de ATZ em $\mathrm{CaCl}_{2}$ 0,01 $\mathrm{mol} \mathrm{L}^{-1}$, preparada a partir da ATZ comercial. Os testes de liberação foram realizados em duplicata em frasco plástico escuro com tampa contendo $100 \mathrm{~mL}$ de solução de $\mathrm{CaCl}_{2} 0,01 \mathrm{~mol} \mathrm{~L}^{-1}$ e uma determinada massa de formulação de ATZ. A massa de formulação empregada foi calculada considerando-se que a liberação total de ATZ não ultrapassasse $15 \mathrm{mg} \mathrm{L}^{-1}$, que representa a concentração máxima permitida para que a linearidade da Lei de Beer-Lambert seja respeitada para a ATZ neste meio, no comprimento de onda de 222 $\mathrm{nm}$. As suspensões foram agitadas em agitador horizontal durante $360 \mathrm{~min}$ à temperatura ambiente e alíquotas de $2 \mathrm{~mL}$ foram coletadas periodicamente neste intervalo para determinação da absorbância. Após esse tempo as medidas foram suspensas devido à estabilização da quantidade liberada. Após cada retirada de alíquota o volume era reconstituído adicionando-se igual volume de solução de $\mathrm{CaCl}_{2}$ $0,01 \mathrm{~mol} \mathrm{~L}^{-1}$. Para o cálculo de cada concentração final, empregou-se um fator de correção para corrigir a diluição devida a cada coleta, multiplicando-se o mesmo à concentração determinada pela curva padrão. As coletas foram realizadas a cada 5 min nos primeiros 30 min, e a seguir a cada 30 min.

A proporção de $\mathrm{ATZ}$ liberada $\left(\mathrm{ATZ}_{\mathrm{L}}\right.$ ) foi calculada relacionandose a quantidade liberada em um dado instante pela quantidade total contida na amostra $\left(\mathrm{m}_{\text {ATZ }}\right)$ (Equação 4$)$

$$
A T Z_{L}(\%)=\frac{[A T Z]\left(m g \cdot L^{-1}\right) \times V(L)}{m_{A T Z}(m g)} \times 100
$$

onde [ATZ] é a concentração de ATZ na alíquota determinada pela curva padrão e corrigida pelo fator de diluição, e V é o volume da solução de análise $(0,1 \mathrm{~L})$.

Os dados obtidos na liberação foram analisados aplicando-se modelos matemáticos usualmente empregados em liberação de fármacos: ordem zero, primeira e segunda ordens, Higuchi (\%diss $\left.=\mathrm{kt}^{0,5}\right)$ e Korsmeyer-Peppas $\left(\%\right.$ diss $\left.=a t^{n}\right)$, onde \%diss corresponde à porcentagem de ATZ liberada no tempo t. No ajuste dos modelos foram considerados os pontos de liberação até $70 \%{ }^{23}$

\section{Isoterma de sorção da ATZ em amostras de solo}

A sorção da ATZ foi realizada em amostras de horizonte A de solos coletados sob campo nativo de um Latossolo Bruno (Vacaria, RS), Latossolo Vermelho (Augusto Pestana, RS) e Latossolo Vermelho-Amarelo (Brasília, DF), cujas características químicas foram estudadas previamente. ${ }^{24}$

Para obtenção das isotermas de sorção foram empregadas $1,0 \mathrm{~g}$ de solo e $50 \mathrm{~mL}$ de solução de ATZ granulada e de SGATZ150 nas concentrações de $0,5,10,20,30 \mathrm{mg}$ ATZ L-1 em meio de $\mathrm{CaCl}_{2}$ $0,01 \mathrm{~mol} \mathrm{~L}^{-1}$. Visando simular as condições de aplicação do produtor a campo, as soluções adicionadas em cada ponto da isoterma (duplicata) foram preparadas separadamente a partir de uma massa de SGATZ ou de ATZGRAN pesadas para a determinada concentração, sem agitação prévia para liberação do ativo. Após 24 h de agitação em contato com o solo, a suspensão foi centrifugada (3000 rpm) e o sobrenadante filtrado em filtro de papel qualitativo para eliminar partículas suspensas. As soluções contendo ATZ foram concentradas em cartuchos SPE a um volume de $10 \mathrm{~mL}$ e a concentração determinada por cromatografia gasosa. $\mathrm{O}$ procedimento empregado baseou-se na técnica utilizada por Kleinschmitt. ${ }^{25}$

Na quantificação do herbicida nos extratos foi utilizado o método de padronização externa. Para cada solo e formulação testados foram obtidas curvas de calibração separadamente, a partir de 5 soluções de concentração conhecida no intervalo entre 0 a $30 \mathrm{mg} \mathrm{L}^{-1}$, utilizadas na sorção.

A quantificação de ATZ foi feita em cromatógrafo em fase gasosa Shimadzu GC 17A, equipado com injetor split/splitless, detector de ionização de chama (FID) e coluna capilar SPB-5 [poly (5\%diphenyl95\%dimethylsiloxane)], $30 \mathrm{~m}$ x 0,25 $\mathrm{mm}$ e 0,25 $\mu \mathrm{m}$. As análises foram realizadas em temperatura programada iniciando-se em 130 ${ }^{\circ} \mathrm{C}$ durante $1 \mathrm{~min}$ e, a seguir, uma rampa de aquecimento na seguinte ordem: $10^{\circ} \mathrm{C} / \mathrm{min}-190^{\circ} \mathrm{C}, 15^{\circ} \mathrm{C} / \mathrm{min}-280^{\circ} \mathrm{C}$, e, por fim, $1 \mathrm{~min}$ em temperatura constante de $280^{\circ} \mathrm{C}$. A temperatura do injetor foi de 250 ${ }^{\circ} \mathrm{C}$ e o detector de $295^{\circ} \mathrm{C}$. O gás de arraste utilizado foi hidrogênio 5.0 (White Martins). O volume de injeção foi de $1 \mu \mathrm{L}$ de amostra. Cada amostra foi injetada duas vezes e no caso de divergência dos resultados, uma terceira injeção foi realizada.

A quantidade de atrazina sorvida no solo $\left(Q_{S}\right)$ foi calculada pela diferença entre a concentração inicial adicionada ao solo e a concentração final em equilíbrio (Equação 5).

$Q s\left(m g\right.$ ATZ $\cdot g^{-1}$ solo $)=\frac{\left[C_{o}-C_{e}\right]\left(m g A T Z \cdot L^{-1}\right) x V(L)}{m_{\text {solo }}(g)}$

onde $\mathrm{C}_{0}$ é a concentração inicial, $\mathrm{C}_{\mathrm{e}}$ é a concentração final em equilíbrio, $\mathrm{V}$ é o volume da solução adicionada ao solo e $\mathrm{m}_{\text {solo }}$ é a massa do solo. As isotermas foram traçadas considerando as médias aritméticas das duplicatas dos valores de $\mathrm{Q}_{\mathrm{S}}$ e $\mathrm{C}_{\mathrm{e}}$.

Foi calculada a regressão linear para os pontos das isotermas, determinando-se a sua probabilidade estatística de erro segundo o teste de Fischer. Para o cálculo do índice Kd de cada sistema sorbatosorvente calculou-se uma regressão linear separadamente para cada série de pontos de uma duplicata. $\mathrm{O}$ valor de $\mathrm{Kd}$ foi obtido pela média aritmética dos parâmetros angulares de cada regressão, sendo calculado o respectivo desvio padrão. Procedimento semelhante foi realizado para o índice $\mathrm{K}_{\mathrm{oc}}$ que expressa o índice $\mathrm{K}_{\mathrm{d}}$ por unidade de Carbono do solo (Equação 6):

$K_{o c}\left(L . \mathrm{kg}^{-1} \mathrm{C}\right)=\frac{\left[K_{d}\left({\left.\mathrm{~L} . \mathrm{kg}^{-1} \text { solo }\right)}^{-1}\right.\right.}{C\left(\mathrm{~g} \cdot \mathrm{kg}^{-1} \text { solo }\right)} \times 1000$

\section{RESULTADOS E DISCUSSÃO}

\section{Caracterização das formulações e do produto comercial}

Os teores de $\mathrm{C}$ e de $\mathrm{N}$ nas formulações aumentaram gradualmente com o aumento da quantidade de ATZ adicionada à síntese, e a concentração de ATZ nas formulações variou de 55,0 a 188,0 mg $\mathrm{ATZ} \mathrm{g}^{-1} \mathrm{SiO}_{2}$ (Tabela 1).

Os rendimentos das sínteses situaram-se entre 58 e $68 \%$, sendo os maiores valores observados nas concentrações intermediárias de ATZ. Estes valores são inferiores aos usualmente encontrados para formulações de fármacos encapsulados em xerogéis. ${ }^{26} \mathrm{No}$ presente estudo, provavelmente, devido à baixa solubilidade da ATZ em meio aquoso $\left(33 \mathrm{mg} . \mathrm{L}^{-1}\right),{ }^{27}$ associado à presença de aditivos e à secagem prévia do produto comercial que prejudicam sua ressolubilização, parte da mesma fica retida nas paredes do recipiente por ocasião da agitação da suspensão, previamente à adição ao catalisador.

Nos produtos comerciais (ATZ comercial e ATZ granulada) a concentração de herbicida foi inferior a $100 \%$, confirmando a pre- 
sença de estabilizantes e outros excipientes cujas concentrações não são descritas nos rótulos. A pureza da amostra de princípio ativo foi confirmada pela concentração de ATZ determinada (Tabela 1).

Na micrografia da amostra de sol-gel puro, são visíveis micropartículas sem forma organizada e de superfície irregular (Figura 1a). Na superfície da amostra de ATZ granulada, identificam-se grânulos comparativamente maiores e com formas irregulares (Figura 1b). Por outro lado, nas formulações (Figuras 1c, d, e e f) ocorrem estruturas mais cristalinas em forma de agulhas. Nestas, a concentração de ATZ é maior do que no restante da superfície de acordo com os resultados obtidos por EDS através de análises pontuais dos dois tipos de estruturas visualizados nas micrografias. A porcentagem atômica de cloro encontrada nas estruturas aciculares foi de 11 a 15 vezes maior do que a das regiões onde não se visualiza este tipo de estrutura. Comparando-se as micrografias de xerogéis, observa-se que os cristais tendem a ser maiores com o aumento da concentração de ATZ .

A área superficial das formulações aumentou com o incremento da quantidade de ATZ adicionada à síntese (Tabela 1), aproximando-

(a)

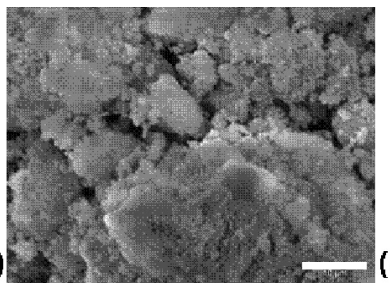

(c)

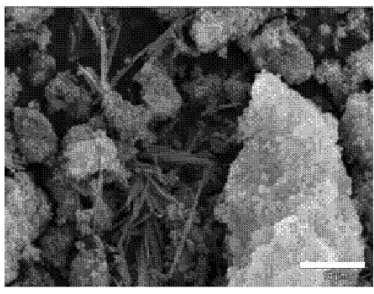

(e)

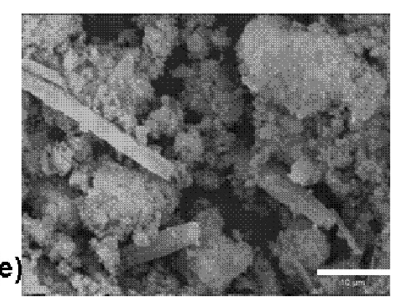

(d)
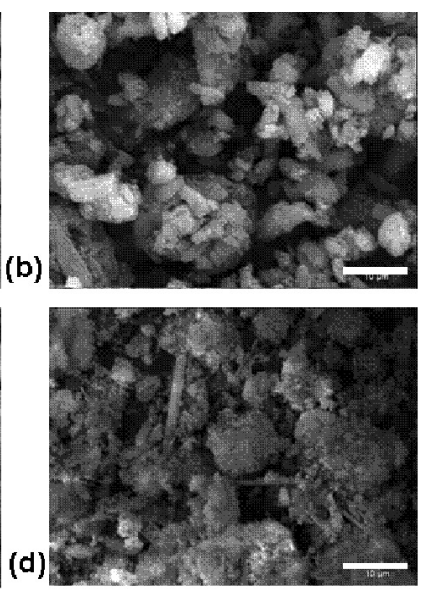

Figura 1. Micrografias de microscopia eletrônica de varredura (MEV) (barra $=10 \mu \mathrm{m})$ das amostras de sol-gel (a), ATZ granulada (b), e das formulações SGATZ150 (c), SGATZ200 (d), SGATZ300 (e) e SGATZ500 (f) se do valor encontrado para o xerogel puro $\left(145 \pm 8 \mathrm{~m}^{2} \mathrm{~g}^{-1}\right)$. Este comportamento pode indicar que com o aumento da concentração do herbicida na formulação, maior é a tendência de aglomeração em cristais, diminuindo assim a dispersão do ativo nos poros da matriz.

Nas análises de DSC, as amostras de ATZ comercial, ATZ granulada e ATZ pura apresentaram um único pico endotérmico em $177,5^{\circ} \mathrm{C}$, correspondendo à temperatura de fusão do herbicida. ${ }^{28}$ Os termogramas das formulações apresentaram comportamento térmico semelhante ao da ATZ pura, não havendo deslocamento da temperatura de fusão. Este resultado indica que a ATZ não interage quimicamente com o xerogel e está fisicamente distribuída no mesmo. A entalpia específica de fusão $\left(\Delta \mathrm{H}_{\text {fusão }}\right)$ da amostra de ATZ pura foi de 38,2 cal g-1 ATZ enquanto nas amostras comerciais os valores foram inferiores (Tabela 1). Nas formulações o $\Delta \mathrm{H}_{\text {fusão }}$ aumenta com o teor de ATZ contido nas mesmas, tendendo para o valor da ATZ pura (Tabela 1). A correlação observada entre $\Delta \mathrm{H}_{\text {fusão }}$ e a massa de ATZ presente na formulação $(\mathrm{R}=0,98, \mathrm{P}<1 \%$ ) (Figura $1 \mathrm{~S}$, Material Suplementar) indica que com o aumento da proporção de ATZ ocorre saturação da matriz do xerogel e o herbicida tende a apresentar-se em cristais maiores. Esse comportamento corrobora os resultados da ASE e os obtidos pela análise de DSC.

Nos espectros de FTIR da ATZ pura, comercial e granulada (Figura 2, espectros a, b e c, respectivamente) as principais bandas de absorção identificadas e suas atribuições ${ }^{29}$ foram: $3262 \mathrm{~cm}^{-1}$, deformação axial simétrica e assimétrica de N-H; 2960 e $2850 \mathrm{~cm}^{-1}$, estiramento de C-H de alifáticos; 1628 e $1550 \mathrm{~cm}^{-1}$ deformação da ligação $\mathrm{C}=\mathrm{C}$ e $\mathrm{C}=\mathrm{N}$.

No espectro do SG foi identificada uma banda larga em 1140 $\mathrm{cm}^{-1}$, característica do estiramento Si-O-Si e uma banda em 3500 $\mathrm{cm}^{-1}$ característica dos grupos silanóis em ponte (Figura 2S, Material Suplementar). A ausência de banda na região de $3740 \mathrm{~cm}^{-1}$ indica que grupos silanóis livres não estão presentes. A banda em $1630 \mathrm{~cm}^{-1}$ é referente a uma banda de sobretom da molécula de água. ${ }^{30}$ No espectro da formulação SGATZ150 (Figura 2, espectro a), que apresentou a maior dispersão de ATZ na matriz de sol-gel comparativamente às outras formulações, foram identificadas bandas características do herbicida: 3260, 2940, 1619 e $1553 \mathrm{~cm}^{-1}$. O fato de não haver deslocamento relevante das bandas dos grupos $\mathrm{N}-\mathrm{H}$ e C-H sugere que não houve formação de ligação química entre estes grupos da ATZ e o SG na síntese do SGATZ, corroborando os resultados obtidos por DSC. Nos espectros das outras formulações (Figura 2, espectros b, c e d) este comportamento se mantém. Com o aumento da concentração de herbicida na formulação aumentam os domínios de cristais isolados de ATZ segundo dados de MEV e, consequentemente, sua contribuição para as bandas de absorção. Porém, devido ao fato de a formulação SGATZ150 apresentar o mesmo padrão de espectro que as restantes

Tabela 1. Concentração de ATZ nas formulações sintetizadas e produtos comerciais, rendimento (R) das sínteses, $\Delta \mathrm{H}_{\text {fusão }}$ das formulações e das amostras comerciais de ATZ, área superficial específica (ASE) das formulações (médias de duplicatas), constantes $a, n$ e de correlação (r) para o modelo de Korsmeyer-Peppas aplicado à cinética de liberação de ATZ das formulações sintetizadas

\begin{tabular}{|c|c|c|c|c|c|c|c|}
\hline Amostra & $\begin{array}{c}\mathrm{ATZ} \\
\mathrm{mg} \mathrm{g}^{-1} \mathrm{SiO}_{2}\end{array}$ & $\begin{array}{l}\mathrm{R} \\
\% \\
\end{array}$ & $\begin{array}{l}\text { ASE } \\
m^{2} \mathrm{~g}^{-1}\end{array}$ & $\begin{array}{c}\Delta \mathrm{H} \text { fusão } \\
\text { cal.g-1 ATZ }\end{array}$ & $a$ & $n$ & $r$ \\
\hline SGATZ150 & 55,5 & 57,7 & $82 \pm 7$ & 18,0 & 2,96 & 0,3313 & 0,998 \\
\hline SGATZ200 & 87,5 & 68,3 & $60 \pm 6$ & 25,0 & 3,55 & 0,2314 & 0,999 \\
\hline SGATZ300 & 123,0 & 63,9 & $105 \pm 8$ & 26,8 & 3,72 & 0,1808 & 0,995 \\
\hline SGATZ500 & 188,3 & 58,8 & $150 \pm 8$ & 36,3 & 3,63 & 0,1940 & 0,998 \\
\hline ATZ granulada & 921,4 & n.d & n.d & 37,6 & 2,60 & 0,3823 & 0,987 \\
\hline ATZ comercial & 862,0 & n.d & n.d & 33,0 & n.d. & n.d & n.d \\
\hline ATZ pura & 999,9 & n.d & n.d & 38,2 & n.d & n.d & n.d \\
\hline
\end{tabular}




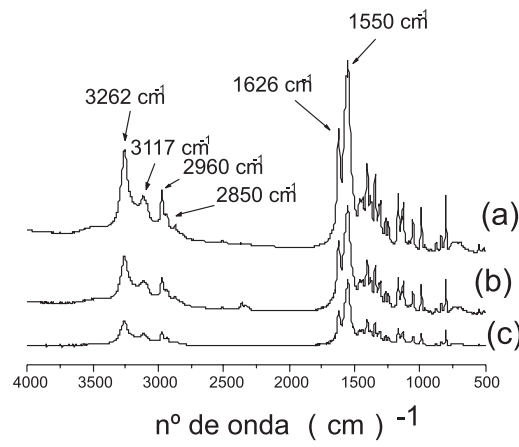

(a)

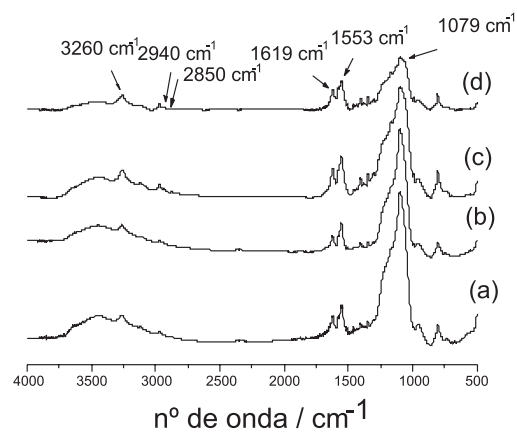

(b)

Figura 2. Espectros de FTIR das amostras de ATZ (a): amostra pura (a), ATZ comercial (b) e ATZ granulada (c); Espectros de FTIR das formulações $(\boldsymbol{b})$ : SGATZ150 (a), SGATZ200 (b), SGATZ150 (c), SGATZ150 (d)

não é possível distinguir as contribuições dos dois tipos de domínio nas formulações mais concentradas.

\section{Cinética de liberação de ATZ das formulações em meio aquoso}

Tanto nas formulações como na amostra granulada, a proporção de ATZ liberada da fase sólida cresce ao longo do tempo tendendo à estabilização entre 180 e 360 min com valores entre 75 e $96 \%$ da quantidade presente na formulação. (Figura 3). Aos $200 \mathrm{~min}$, as formulações SGATZ200, SGATZ300 e SGZTZ500 liberaram aproximadamente $95 \%$ do herbicida, enquanto a formulação SGATZ150 liberou $75 \%$ e a forma granulada, $85 \%$.

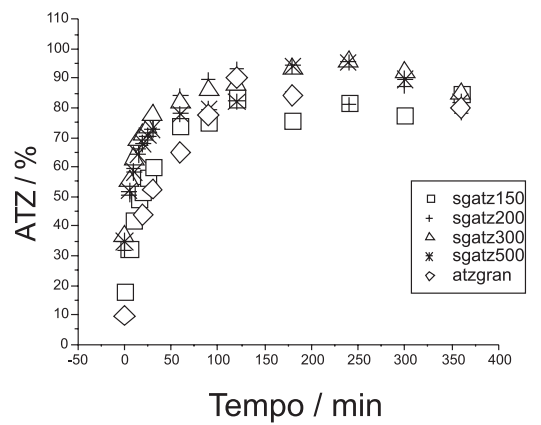

Figura 3. Proporção de ATZ liberada das formulações em meio de $\mathrm{CaCl}_{2}$ $0,01 \mathrm{~mol} \mathrm{~L}^{-1}$ em função do tempo

Entre os modelos matemáticos testados aos dados de liberação, o que melhor se ajustou aos dados experimentais foi o modelo de Korsmeyer-Peppas, cujos coeficientes de correlação (Tabela 1) foram superiores aos dos outros modelos (dados não mostrados). No modelo de Korsmeyer-Peppas, "a" é uma constante que incorpora características estruturais e geométricas do sistema de liberação, e "n" permite inferir sobre o mecanismo de liberação da substância. ${ }^{31}$

Para sistemas esféricos, $\mathrm{n}=0,43$ indica que o mecanismo de liberação é dependente da difusão do ativo, seguindo a segunda Lei de Fick. Se $n=0,85$, a liberação da substância é controlada pela ação do solvente na matriz, seguindo o modelo de cinética de ordem zero. Para valores intermediários de "n", a difusão é dita anômala, e o mecanismo de liberação corresponde à difusão do ativo através da matriz e ao inchamento/erosão da matriz por ação do solvente.

No presente trabalho os valores de "n" são inferiores a 0,4 (Tabela 1), e no caso específico das formulações estes coeficientes tendem a diminuir com o aumento da proporção de ATZ na amostra. Provavelmente nos testes de liberação em meio aquoso da amostra de ATZ granulada e de SGATZ150 está ocorrendo predominância do fenômeno de dissolução sobre o de difusão. Com o aumento da proporção de ATZ nas formulações, cujos cristais tendem a ser maiores (Figura 1), a dissolução domina a liberação.

Os resultados dos testes realizados, ainda que não reproduzam as condições naturais do solo, permitem avaliar e comparar o comportamento da liberação do herbicida entre as formulações, sob condições controladas e de estresse mecânico semelhante, que desloca o equilíbrio no sentido da liberação. Portanto, considerando-se os valores da Tabela 1 e a Figura 3, infere-se que apenas a formulação de menor concentração apresenta comportamento semelhante ao do produto granulado.

\section{Isoterma de sorção da ATZ em solos}

Os testes de sorção foram realizados com a formulação SGATZ150 devido esta ter apresentado a maior dispersão de ATZ na matriz e menor quantidade de herbicida liberado até 200 min, entre as formulações estudadas. Para fins de comparação foi realizada a sorção de ATZ na forma granulada.

Os coeficientes de correlação para o ajuste linear das isotermas de sorção variaram entre 0,84 e 0,98 , e a significância estatística entre 2 e $16 \%$ (Figura 4). A elevada probabilidade de erro observada em algumas isotermas possivelmente está relacionada à heterogeneidade da amostra sólida, o que pode levar a uma super- ou sub-estimação da quantidade de atrazina no momento da pesagem para preparo da solução de cada ponto da isoterma. No presente estudo optou-se em preparar a solução de ATZ simulando seu uso pelo produtor, ou seja, não houve agitação da solução visando solubilização do herbicida previamente à sua adição ao solo.

Os coeficientes de distribuição $\mathrm{K}_{\mathrm{d}}$ situaram-se entre 4,0 e 5,4 L.kg ${ }^{1}$ solo para SGATZ150 e entre 12 e 14,6 L.kg-1 solo para ATZGRAN (Tabela 2). No Latossolo Bruno e no Latossolo Vermelho Kd para a formulação foi menor do que para ATZGRAN, sugerindo que nestes solos a ATZ na forma granulada é mais retida pelo solo do que na forma de xerogel.

Os valores de $\mathrm{K}_{\mathrm{d}}$ para SGATZ150 decresceram na ordem Latossolo Vermelho Amarelo > Latossolo Bruno > Latossolo Vermelho, enquanto que para a forma granulada não houve diferença relevante entre os solos.

Analogamente ao ocorrido com o $\mathrm{K}_{\mathrm{d}}$, os valores de $\mathrm{K}_{\mathrm{oc}}$ do xerogel SGATZ150 foram inferiores aos da ATZ granulada para Latossolo Bruno e para Latossolo Vermelho, confirmando que a ATZ na forma de xerogel apresenta menor afinidade pela matéria orgânica do que na forma granulada neste dois sorventes. $\mathrm{O}$ índice de $\mathrm{K}_{\mathrm{oc}}$ para SGATZ150 aumentou na ordem: Latossolo Bruno $\approx$ Latossolo Vermelho $<$ Latossolo Vermelho Amarelo, enquanto para ATZGRAN os valores não diferiram relevantemente entre si. 
Tabela 2. Teores de carbono e de argila, área superficial específica (ASE), coeficientes de distribuição $K_{d}$ e $K_{o c}$ para atrazina nas formas SGATZ150 e ATZGRAN sorvidas em Latossolo Bruno, Latossolo Vermelho e Latossolo Vermelho Amarelo

\begin{tabular}{|c|c|c|c|c|c|c|c|}
\hline \multirow{2}{*}{ Solo } & \multirow{2}{*}{$\mathrm{C} / \mathrm{g} \mathrm{kg}^{-1}$} & \multirow{2}{*}{ Argila / $\mathrm{g} \mathrm{kg}^{-1}$} & \multirow{2}{*}{$\mathrm{ASE} / \mathrm{m}^{2} \mathrm{~g}^{-1}$} & \multicolumn{2}{|c|}{ SGATZ150 } & \multicolumn{2}{|c|}{ ATZGRAN } \\
\hline & & & & $\mathrm{K}_{\mathrm{d}} / \mathrm{L} \mathrm{kg}^{-1}$ & $\mathrm{~K}_{\mathrm{oc}} / \mathrm{L} \mathrm{kg}^{-1}$ & $\mathrm{~K}_{\mathrm{d}} / \mathrm{L} \mathrm{kg}^{-1}$ & $\mathrm{~K}_{\mathrm{oc}} / \mathrm{L} \mathrm{kg}^{-1}$ \\
\hline $\begin{array}{l}\text { Latossolo } \\
\text { Bruno }\end{array}$ & 35,1 & 524 & 63,6 & $5,4 \pm 1,1$ & $153,8 \pm 32,2$ & $12,0 \pm 4,5$ & $341,7 \pm 129,1$ \\
\hline $\begin{array}{l}\text { Latossolo } \\
\text { Vermelho }\end{array}$ & 22,5 & 625 & 47,3 & $4,0 \pm 0,8$ & $178,2 \pm 35,8$ & $14,2 \pm 1,0$ & $633,6 \pm 46,2$ \\
\hline $\begin{array}{l}\text { Latossolo } \\
\text { Vermelho } \\
\text { Amarelo }\end{array}$ & 26,2 & 633 & 33,5 & $10,7 \pm 0,4$ & $407,8 \pm 15,9$ & $14,6 \pm 7,5$ & $559,7 \pm 285,8$ \\
\hline
\end{tabular}
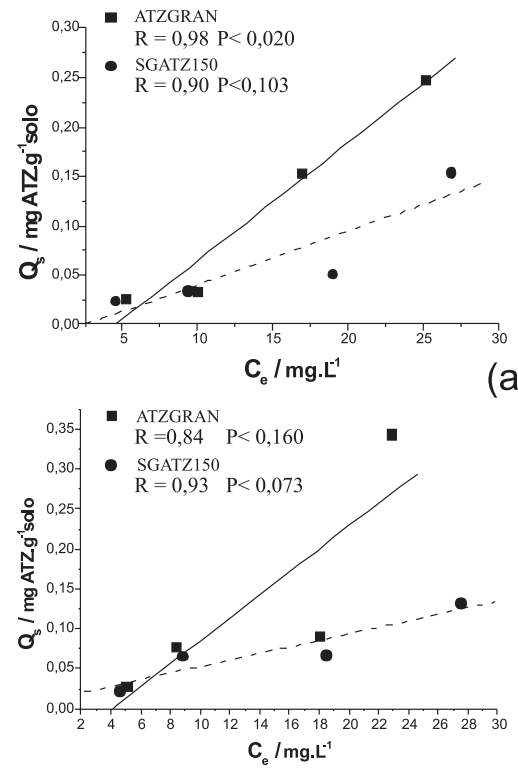

(b)

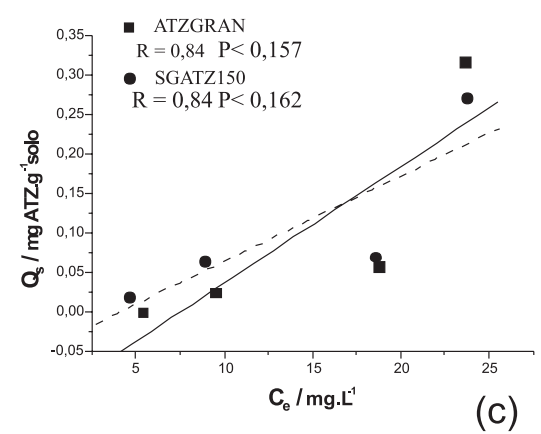

Figura 4. Isoterma de sorção de ATZ na forma (---) SGATZ150 e ( - )ATZGRAN no Latossolo Bruno (a), no Latossolo Vermelho (b) e no Latossolo Vermelho - Amarelo (c). (Probabilidade de erro segundo teste de Fischer)

Os maiores valores de $\mathrm{K}_{\mathrm{d}}$ e $\mathrm{K}_{\mathrm{oc}}$ de SGATZ150 observados para o Latossolo Vermelho Amarelo comparativamente aos outros dois solos, não se relacionaram com suas características (Tabela 2).

\section{CONCLUSÕES}

Os rendimentos obtidos nas formulações de liberação controlada de atrazina situaram-se em torno de $60 \%$, e podem ser considerados satisfatórios. Quanto menor a concentração do herbicida na formulação, mais dispersa fisicamente encontra-se o mesmo na matriz. A liberação do herbicida da formulação para o meio é controlada predominantemente pelo fenômeno de dissolução, que se torna o principal mecanismo para formulações com concentrações de ATZ mais elevadas, onde os cristais do ativo são maiores. Das formulações testadas apenas a de menor concentração de ATZ liberou menor quantidade de herbicida do que a forma granulada em aproximadamente $3 \mathrm{~h}$.

O comportamento sortivo observado para os dois tipos de sorventes nos três solos avaliados, ainda que resultante de situações diversas às condições de sorção no solo, permite inferir que a ATZ na forma de xerogel tende a apresentar menor afinidade pelo solo do que na forma granulada.

A partir destes resultados preliminares, estudos de incubação de solo com a fórmula SGATZ150 estão previstos para se averiguar se, em ausência de estresse mecânico, o comportamento da cinética de liberação desta formulação se mantém inferior ao da forma granulada. Este fato associado a sua menor afinidade pelo solo poderia propiciar uma maior eficiência de absorção do ativo pela planta alvo. No entanto, ainda que nossos dados sejam promissores, nada pode ser afirmado até o momento quanto à eficiência agronômica da atrazina na forma de xerogel.

\section{AGRADECIMENTOS}

L.G. de Ávila agradece à CAPES pela bolsa de mestrado. A. R. Pohlmann e D. P. Dick ao CNPq pelas bolsas de produtividade em pesquisa. Os autores agradecem ao Prof. Dr. E. Benvenutti pela disponibilidade do equipamento de determinação da ASE e ao Prof. Dr. P. Brenner pelas análises elementares, ambos da UFRGS.

\section{REFERÊNCIAS}

1. http://www.conab.gov.br, acessada em Abril 2009.

2. Van Maanen, J. M.; De Vaan, M. A.; Veldstra, A. W.; Hendry, W. P.; Environ. Monit. Assess. 2001, 72, 95.

3. Cerejeira, M. J.; Viana P.; Batista, S.; Pereira,T.; Silva, E.; Valério, M. J.; Silva, A.; Ferreira, M.; Silva-Fernandes, A. M.; Water Res. 2003, 37, 1055 .

4. Boyd, R. A.; Sci. Total Environ. 2000, 248, 241.

5. US Environmental Protection Agency; EPA draft final list of recommendation for chemicals in the National Survey for Pesticides in Groudwate, Chem. Regul. Rep. 1985, 9, 1033.

6. Graymore, M.; Stagnitti, F.; Allinson, G.; Environ. Int. 2001, 26, 483.

7. Lavorenti, A.; Prata, F.; Regitano, J. B.; Comportamento de pesticidas em solos, Fundamentos. Tópicos Especiais em Ciência do Solo, Ed. da UFV: Viçosa, 2003.

8. Triegel, E. K.; Guo, L. Em Mecanisms of pesticide movement into groundwater; Honeycutt, R. C.; Schalacker, D. J., eds.; CRC Press: Boca Raton, 1994.

9. Sparks, D. L.; Sorption phenomena on soils, Environmental Soil Chemistry: Academic Press, 1995. 
10. Weber, J. B.; Wilkerson, G. G.; Linker, H. M.; Wilcut, J. W.; Leidy, R. B.; Senseman, S.; Witt, W. W.; Barret, M.; Vencill, W. K.; Shaw, D. R.; Mueller, T. C.; Miller, D. K.; Brecke, B. L.; Talbert, R. E.; Peeper, T. F.; Weed Sci. 2000, 48, 75.

11. Pohlmann, A. R.; Cruz, L.; Soares, L. U.; Dalla Costa, T.; Mezzalira, G.; Silveira, N. P.; Guterres, S. S.; Int. J. Pharmac. 2006, 313, 198.

12. Böttcher, H.; Slowik, P.; Süß, W.; J. Sol-gel Sci. Technol. 1998, 13, 277.

13. Mah, S. K.; Chung, I. J.; J. Non-Crystalline Solids 1995, 183, 252.

14. Sieminska, L.; Buntner, B.; Woznica, A.; Zerda, T. W.; Euro. J. Pharm. Sci. 1996, 4, 155.

15. Dailey Jr, O. D.; Dowler, C. C.; Mullinix Jr, B. G.; J. Agric. Food Chem. 1993, 41, 1517.

16. Pepperman, A. B.; Kuan, J. W.; J. Controlled Release 1995, 34, 17.

17. Prado, A. G. S.; Airoldi, C.; Pest Manag. Sci. 2000, 56, 419.

18. Prado, A. G. S.; Airoldi, C.; J.Colloid Interface Sci. 2001, 236, 161.

19. Prado, A. G. S.; Airoldi, C.; Thermochim. Acta 2002, 394, 163.

20. Prado, A. G. S.; Airoldi, C.; Green Chem. 2002, 4, 288

21. Benvenutti, E. V.; Pavan, F. A.; Gushikem, Y.; Moro, C. C.; Costa, T. M.; Colloid Polymer Science 2003, 281, 173.
22. Guterres S. S.; Raffin, R. P.; Colomé, L. M.; Pohlmann, A.R.; Eur. J. Pharma. Biopharmac. 2006, 63, 198.

23. Korsmeyer, R. W.; Gurny, R.; Doelker, E.; Buri, P.; Peppas, N. A.; Int. J. Pharmaceutics 1983, 15, 25.

24. Dick, D. P.; Gonçalves, C. N.; Dalmolin, R. S. D.; Dick, D. P.; Knicker, H.; Klamt, E.; Kögel-Knabner, I.; Simões, M. L.; Martin-Neto, L.; Geoderma 2005, 124, 319.

25. Kleinschmitt, A. R. B.; Dissertação de Mestrado, Universidade Federal do Rio Grande do Sul, Brasil, 2003.

26. Fonseca, L. S.; Silveira, R. P.; Deboni, A. M.; Benvenutti, E. V.; Costa, T. H.; Guterres, S.; Pohlmann, A. R.; Int. J. Pharmaceutics 2008, 358, 292.

27. Vidal, R. A.; Merotto Jr., A.; Herbicidologia, Evangraf: Porto Alegre, 2001.

28. http://www.nist.gov/srd/online.html, acessada em Outubro 2007.

29. Silverstein, R. M.; Identificação espectrométrica de compostos orgânicos, $6^{\mathrm{a}}$ ed., LTC: Rio de Janeiro, 2000.

30. Benvenutti, E.; Azolin, D. R.; Moro, C. C.; Costa, T. M. H.; J. NonCryst. Solids 2004, 337, 201.

31. Costa, P.; Lobo, J. M. S.; Euro. J.Pharmac. Sci. 2001, 13, 123. 


\section{FORMULAÇÕES DE ATRAZINA EM XEROGÉIS: SÍNTESE E CARACTERIZAÇÃo}

Letícia Gomes de Ávila*, Simone Benvenuti Leite e Deborah Pinheiro Dick

Departamento de Físico-Química, Instituto de Química, Universidade Federal do Rio Grande do Sul, CP 15003, 91501-970 Porto Alegre - RS, Brasil

Adriana Raffin Pohlmann

Departamento de Química Orgânica, Instituto de Química, Universidade Federal do Rio Grande do Sul, CP 15003, 91501-970 Porto Alegre - RS, Brasil

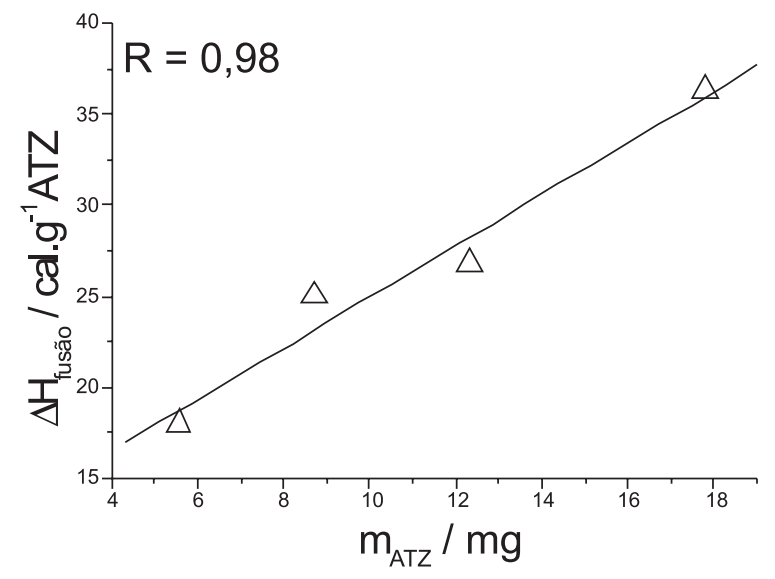

Figura 1S. Relação entre $\Delta H_{\text {fusão }}$ e massa de ATZ $\left(m_{\text {ATZ }}\right)$ contida nas formulações

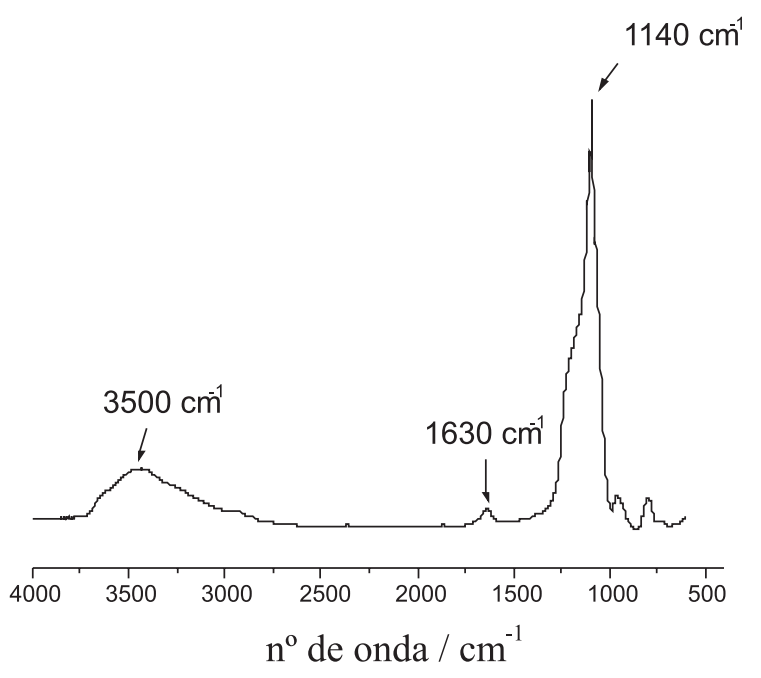

Figura 2S. Espectro de FTIR da amostra de sol-gel puro (SG)

*e-mail: leticia@iq.ufrgs.br 